\title{
Avaliação de uma nova metodologia de irradiação de mama esquerda na fase de inspiração
} Evaluation of a new irradiation left breast method in the inspiration phase

\author{
Laura E. da Silva ${ }^{1}$, Leonardo P. da Silva² ${ }^{2}$ Rafael G. Gullo ${ }^{3}$, Diogo A. V. Ferreira ${ }^{3}$ \\ ${ }^{1}$ Instituto Nacional de Câncer, Rio de Janeiro, Brasil \\ ${ }^{2}$ Departamento de Física Médica, Intituto Nacional de Câncer, Rio de Janeiro, Brasil \\ ${ }^{3}$ Departamento de Radioterapia, Instituto Nacional de Câncer, Rio de Janeiro, Brasil
}

\begin{abstract}
Resumo
A radioterapia é a principal abordagem terapêutica para câncer de mama e envolve a exposição significante de coração e pulmões, principalmente nos casos de mama esquerda. A implementação de uma metodologia que reduza a dose nesses órgãos é importante para garantir melhor qualidade de vida à paciente. Este trabalho objetiva,então, avaliar redução de dose cardíaca e pulmonar quando se realiza o planejamento radioterápico considerando apenas a fase de inspiração. Três pacientes com mama esquerda presente foram tomografadas durante a respiração livre e sob monitoração respiratória, realizando-se o planejamento com base em cada uma. Os resultados obtidos mostraram uma redução na dose média cardíaca de $53 \%$, em média. Quanto aos volumes pulmonares, obteve-se uma redução de, em média, $44,6 \%$ e $51 \%$ de pulmão esquerdo que recebeu $10 \mathrm{~Gy}$ e $20 \mathrm{~Gy}$, respectivamente. Dessa forma, o estudo mostrou que o emprego do gating respiratório para a radioterapia de mama esquerda pode reduzir doses cardíacas e pulmonares.
\end{abstract}

Palavras-chave: tomografia; radioterapia; neoplasias da mama.

\begin{abstract}
Radiation therapy is the primary therapeutic approach to breast cancer and involves significant exposure heart and lungs, especially in cases of left breast. The implementation of a methodology to reduce the dose in these bodies is important to ensure better quality of life to the patient. This work aims then assess reduced heart and lung dose when performing the radiotherapy planning considering only the inspiration phase. Three patients with breast left present were scanned during free breathing and in respiratory monitoring, performing the planning based on each. The results showed a reduction in mean heart dose of $53 \%$ on average. As for lung volumes, there was obtained a reduction of on average $44.6 \%$ and $51 \%$ of the left lung which received $10 \mathrm{~Gy}$ and $20 \mathrm{~Gy}$, respectively. Thus, the study showed that the use of the respiratory gating left breast radiotherapy reduces heart and lung doses.
\end{abstract}

Keywords: tomography; radiotherapy; breast neoplasms.

\section{Introdução}

O câncer de mama é a neoplasia mais comum entre as mulheres (exceto pele não melanoma) no Brasil e no mundo, respondendo por cerca de $25 \%$ dos novos casos a cada ano ${ }^{1}$. Segundo as estatísticas do Instituto Nacional de Câncer $\left(\mathrm{INCa}^{1}\right.$ para o ano de 2016, esperam-se 57960 novos casos, para o Brasil, sendo mais frequente nas mulheres da região Sul do país. A sobrevida em cinco anos aumentou de $78 \%$ para $87 \%$ entre os períodos de 1995 a 1999 e 2005 a 2009. Tal fato deve-se particularmente à detecção precoce, o que aumenta as chances de tratamento e cura.

O rastreamento por meio da mamografia, atualmente, tem beneficiado pacientes com câncer de mama em estágios iniciais, pois a detecção precoce permitiu substituir a remoção completa da mama (mastectomia radical) pela cirurgia conservadora, mantendo a forma do órgão, melhor cosmese e maior qualidade de vida para as pacientes ${ }^{2}$. A radioterapia adjuvante, nestes casos, tem indicação de eliminar doença microscópica, prevenindo recidiva local. $O$ desenvolvimento tecnológico desta modalidade terapêutica tornou-a uma ferramenta importante para o controle tumoral mamário com mínimas toxicidades. Dentre as técnicas disponíveis para tal tratamento, têm espaço 2D, conformacional (3D-CRT), IMRT (sigla do inglês Intensity Modulated Radiotherapy) e compensação eletrônica ${ }^{3}$. Apesar do avanço tecnológico ao longo dos anos, o moviemnto respiratório da paciente, quando não considerado, pode influenciar o desfecho do tratamento ${ }^{4}$.

Durante a irradiação de mama esquerda, pulmão ipsilateral e coração podem estar englobados nos campos de tratamento, devido à anatomia da paciente. Com a movimentação da parede torácica, estes órgãos variam espacialmente, sendo mais ou menos irradiados. Dessa forma,a dose calculada pelo sistema de planejamento pode ser subestimada ${ }^{5}$.

Expor o coração à radiação envolve o aumento da chance de ocorrência de eventos coronários e mortalidades cardíacas ${ }^{6}$. Darby et $\mathrm{al}^{7}$ demonstrou que a taxa de ocorrência dos principais eventos coronarianos (infarto, revascularização 
miocárdica), após radioterapia de mama, aumenta em $7,4 \%$ a cada aumento de $1 \mathrm{~Gy}$ na dose média do coração. Da mesma forma, a exposição do tecido pulmonar aumenta a incidência de complicações como a pneumonite e fibrose ${ }^{8}$.

O planejamento radioterápico, portanto, para uma paciente submetida à cirurgia conservadora de mama esquerda, deve considerar o padrão respiratório, para minimizar doses de radiação desnecessárias a tecidos sadios ${ }^{4}$. Os recursos para aprimorar a técnica de radioterapia de mama envolvem gating respiratório usando a técnica de inspiração profunda (DIBH, do inglês deep inspiration breath hold $)^{9,10,11,12,13}$. Durante a inspiração, o coração se movimenta posteriormente e inferiormente devido à expansão pulmonar e movimentos diafragmáticos. Então, a distância entre a parede torácica e o coração é máxima na ou próximo do máximo na inspiração profunda. A entrega da radiação apenas nesta fase reduz a área cardíaca exposta a altas doses. Paralelamente, o volume relativo de pulmão irradiado, geralmente, diminui ${ }^{14}$.

Dessa forma, este estudo objetiva avaliar a redução de dose cardíaca e pulmonar com a implementação de gating respiratório em situações de irradiação de mama esquerda em fase de inspiração, a partir dos planejamentos radioterápicos em um sistema de planejamento.

\section{Materiais e Métodos}

Selecionaram-se 3 mulheres com câncer de mama esquerda, que foram submetidas à radioterapia após cirurgia conservadora de mama esquerda. Essas pacientes foram tomografadas sem contraste, utilizando o tomógrafo Brillance ${ }^{\circledR}$ (Phillips, Inc.) adquirindo uma série de imagens durante a respiração livre e outra utilizando a técnica 4D, captando 8 fases do ciclo respiratório da paciente. A figura 1 representa típica curva respiratória obtida durante a aquisição das imagens tomográficas 4D.

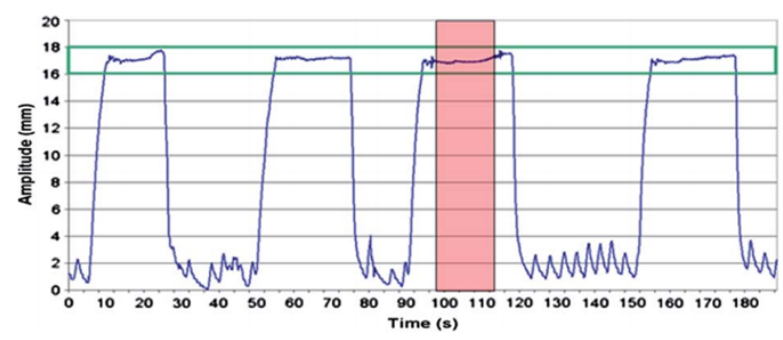

Figura 1. Típica curva respiratória obtida durante a tomografia que considera a fase da inspiração (faixa rosa) com gaps de respiração livre entre cada uma dela ${ }^{15}$.

Esse tomógrafo conta com o bellow, cinto diafragmático, que foi posicionado a $4 \mathrm{~cm}$ do apêndice xifóide, pois a respiração feminina é, predominantemente, torácica.

As pacientes foram posicionadas no breast board em posição supina, com o braço ipsilateral elevado sobre a cabeça para os dois tipos de aquisição de imagem.
O planejamento radioterápico foi realizado para energia de $6 \mathrm{MeV}$, por meio do sistema de planejamento Varian ECLIPSE ${ }^{\circledR}$. A dose de prescrição foi de 50 Gy fracionada em 25 dias consecutivos. Para o planejamento realizado com a tomografia 4D, considerou-se a série de imagens com maior variação da expansividade pulmonar, como se pode observar na figura 2 , onde visualizase uma variação de $9,26 \mathrm{~cm}$ para $12,37 \mathrm{~cm}$, na inspiração. Nessa mesma figura, pode-se verificar ainda o aumento da distância entre o CTV e o coração, durante a inspiração (Figura 2b).

(a)


(b)

Figura 2. Cortes axiais (à direita) e coronais (à esquerda) das tomografias de planejamento na respiração livre (a) e na inspiração profunda (b).

A técnica empregada para cada paciente foi a de campos tangenciais, paralelos à parede torácica e coplanares, como apresentado na figura 3.

(a)

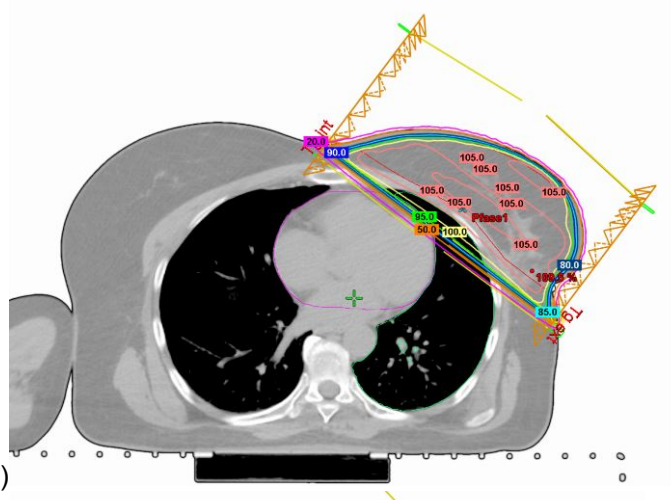

(b)

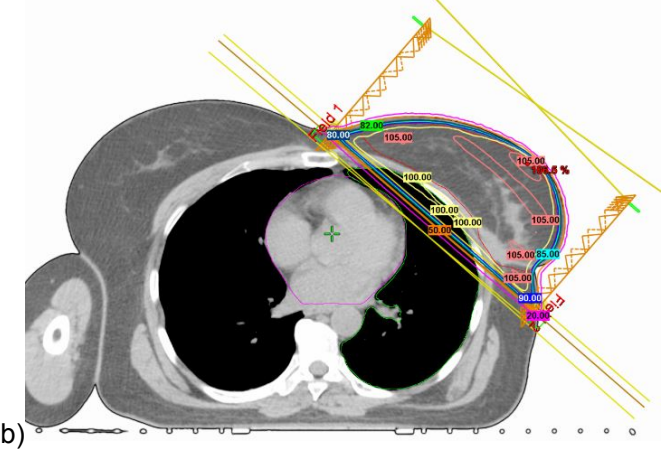

Figura 3. Disposição dos campos de tratamento para o planejamento utilizando tomografia na respiração livre (a) e na inspiração profunda (b). 
Para cada um dos dois planejamentos, respiração livre e da fase da inspiração, cuidou-se para que $95 \%$ da dose de prescrição cobrisse $100 \%$ do CTV de avaliação (do inglês clinical target volume evaluation). O CTV de avaliação foi delineado por um único médico do departamento e consistiu do tecido mamário glandular com margens simétricas de $1 \mathrm{~cm}$.

Para os dois tipos de planejamento, foi utlizada a técnica field-in-field para obter melhor homogeneidade da dose ao longo do volume mamário.

Através da ferramenta histograma de Dosevolume (DVH, do inglês dose-volume histogram) do sistema de planejamento ECLIPSE ${ }^{\circledR}$, obtiveramse os seguintes parâmetros: dose média no coração, volume de pulmão ipsilateral que recebe 10Gy e 20 Gy (V10Gy e V20Gy, respectivamente) e dose média no mesmo. Os valores obtidos desses parâmetros serão comparados entre os planejamentos feitos com a tomografia obtida na respiração livre e na fase da inspiração. Além disso, obteve-se o índice de homogeneidade $(\mathrm{IH})$ para cada planejamento, utilizando a equação 1.

$$
I H=(D 98 \%-D 2 \%) / D 50 \%
$$

sendo D98\%, D2\% e D50\% as doses que englobam $98 \%$, $2 \%$ e $50 \%$ do PTV, respectivamente.

O presente trabalho foi aprovado pelo Comitê de Ética em Pesquisa do Instituto Nacional de Câncer (CEP-INCA) cujo número do protocolo é 1.901.045.

\section{Resultados}

Comparando-se os valores encontrados no DVH para o planejamento que utilizou a tomografia na respiração livre e na inspiração profunda, obtiveram-se as diferenças percentuais apresentadas na tabela 1 .

Também a partir do DVH, obtiveram-se os valores de D2\%, D98\% e D50\% para o PTV, como apresentado na tabela 2 , utilizados para o cálculo de índice de homogeneidade.

Tabela 1. Diferença percentual (\%), para as doses máxima (DDmáx) e média (DDmédia) do coração, para DV20Gy, DV10 Gy e DDmédia do pulmão ipsilateral, entre o planejamento que utiliza a tomografia na respiração livre e na inspiração profunda.

\begin{tabular}{cc|ccc}
\hline & \multicolumn{3}{c}{ Coração } & \multicolumn{3}{c}{ Pulmão Esquerdo } \\
\cline { 2 - 5 } Paciente & $\begin{array}{c}\text { DDmédia } \\
(\%)\end{array}$ & $\begin{array}{c}\text { DV20Gy } \\
(\%)\end{array}$ & $\begin{array}{c}\text { DV10Gy } \\
(\%)\end{array}$ & $\begin{array}{c}\text { DDmédia } \\
(\%)\end{array}$ \\
\hline 1 & $-40,1$ & 69,4 & 78,1 & 40,2 \\
2 & $-65,1$ & $-34,4$ & $-29,6$ & $-27,7$ \\
3 & $-53,5$ & $-67,4$ & $-59,4$ & $-52,8$ \\
\hline
\end{tabular}

Tabela 2. Valores de D2\%, D98\% e D50\% utilizados para o cálculo de índice de homogeneidade para cada um dos planejamentos

\begin{tabular}{cccc|cccc}
\hline \multicolumn{4}{c|}{ Respiração livre } & \multicolumn{4}{c}{ Inspiração profunda } \\
\hline D2\% & D98\% & D50\% & \multirow{2}{*}{ IH } & D2\% & D98\% & D50\% & IH \\
(Gy) & (Gy) & (Gy) & & (Gy) & (Gy) & (Gy) & \\
\hline 54,1 & 48,8 & 52,2 & 10,2 & 53,2 & 48,7 & 52,3 & 8,7 \\
53,0 & 48,5 & 51,9 & 8,6 & 53,9 & 49,0 & 52,5 & 9,4 \\
55,0 & 48,8 & 53,3 & 11,7 & 55,2 & 48,8 & 52,6 & 12,1 \\
\hline
\end{tabular}

\section{Discussão}

A partir da tabela 1, pode-se verificar uma redução de, em média, $53 \%$ para a dose média no coração, quando se realiza o planejamento para radioterapia de mama esquerda, apenas na fase da inspiração profunda. Essa redução de dose cardíaca, segundo a literatura, tem impactos significativos na redução do risco de incidência de efeitos colaterais a longo prazo, devido à radiação ${ }^{13}$.

Para o pulmão, o volume que recebe 10 Gy e 20 Gy teve uma diminuição de, em média, 44,5\%, e $52 \%$, em média. Para a primeira paciente, nota-se que ocorreu um aumento dos volumes pulmonares que receberam 10 Gy e $20 \mathrm{~Gy}$, assim como da dose média no mesmo. Isto foi decorrente do volume mamário da paciente ser menor em $37 \%$, quando comparada às demais. Tal fato evidenciou um critério de elegibilidade das pacientes para uma possível indicação de radioterapia de mama com gating respiratório. No entanto, os dados obtidos para as demais pacientes mostrou a possível eficiência da técnica em reduzir o volume pulmonar irradiado, e, concomitantemente, afastando o músculo cardíaco da área de tratamento.

Quanto ao índice de homogeneidade, pode-se observar que a nova técnica utilizada em comparação à convencional da instituição, teve seus valores variando de $8,7 \%$ a $12,1 \%$, os quais são admíssiveis para um planejamento radioterápico de mama.

Em contrapartida aos resultados obtidos, devese avaliar a implementação desta técnica em um setor público, quanto ao fluxo de tratamentos. Realizar radioterapia de mama apenas na fase de inspiração profunda aumentaria significativamente o tempo de permanência da paciente no aparelho. Isto resultaria em uma possível redução do número de casos tratados por dia, o que contribuiria para o aumento das filas de espera.

\section{Conclusões}

Este estudo comparativo entre planejamento radioterápico de mama esquerda utilizando tomografia na respiração livre e na inspiração profunda, mostrou os benefícios da última técnica, pela redução de doses no coração e no pulmão ipsilateral. Isto refletiria na minimização de efeitos tardios como cardiopatias e pneumonites, que afetam diretamente na qualidade de vida das pacientes, que cada vez mais, têm o diagnóstico precoce e, consequentemente, aumento de sobrevida.

Além disso, este trabalho mostrou a necessidade de se definir critérios de elegibilidade das pacientes, que se beneficiariam com a radioterapia de mama esquerda na fase de inspiração, antes da implementação desta técnica de irradiação.

A implementação da técnica avaliada neste trabalho, em um serviço público, deve ser avaliada 
quanto à demanda do serviço, ponderando-se custos-benefícios.

\section{Agradecimentos}

Ao Instituto Nacional de Câncer (INCa) pela disponibilidade para a realização do trabalho.

\section{Referências}

1. Instituto Nacional de Câncer José Alencar Gomes da Silva. Coordenação de Prevenção e Vigilância Estimativa 2016: incidência de câncer no Brasil / Instituto Nacional de Câncer José Alencar Gomes da Silva - Rio de Janeiro: INCA, 2015.

2. Beck RE, Kim L, Yue N J et. al. Treatment techniques to reduce cardiac irradiation for breast cancer patients treated with breast-conserving surgery and radiation therapy: a review.Frontiers in Oncology 2014; 4: 1-10.

3. Jin G-H, Chen L-X, Deng X-W,et. Al. A comparative dosimetric study for treating left-sided breast cancer for small breast size using five different radiotherapy techniques: conventional tangential field, filed-in-field, tangential-IMRT, Multi-beam IMRT and VMAT. Radiation Oncology 2013; 8: 89-97.

4. Mast ME, van Kempen-Harteveld L, Heijenbrok MW et. al. Left-sided breast cancer radiotherapy with and without breath-hold: Does IMRT reduce cardiac dose even further? Radiotherapy and Oncology 2013; 108: 248-253.

5. Johansen S, Vikström J, Hjelstuem MHB et.al. Dose evaluation and risk estimation for secondary cancer in contralateral breast and a study of correlation between thorax shape and dose to organs at risk following tangentially breast irradiation during deep inspiration breathhold and free breathing. Acta Oncologica 2011;50: 563-568

6. Taylor CW, Povall JM, McGale P et. al. Cardiac dose from tangential breast cancer radiotherapy in the year 2006 . Radiation Oncology Biol Phys 2008; 72: 501-507.

7. Darby SC, Ewertz M, McGale P, et. Al. Risk of ischemic heart disease in women after radiotherapy for breast cancer. N Engl J Med 2013; 368: 987-98.

8. Kim HJ, Jang WI, Kim TJ, Kim JH et. al. Radiation-induced pulmonary toxicity and related risk factors in breast cancer. J Breast Cancer 2009; 12: 67-72.

9. Joo JH, Kim SS, Ahn SD, et.al. Cardiac dose reduction during tangential breast irradiation using deep inspiration breath hold: a dose comparison study based on deformable image registration. Radiation Oncology 2015; 10: 264.

10. Mulliez, T., Veldeman, L., Spelleers, B. Heart dose reduction by prone deep inspiration breath hold in left-sided breast irradiation.Radiotherapy and Oncology (2015). vol. 114, 79-84.

11. Smyth LM, Knight KA, Aarons YK. The cardiac dose-sparing benefits of deep inspiration breath-hold in left breast irradiation: a systematic review. Journal of Medical Radiation Sciences 2015; 62: 66-73.

12. Nissen HD, Appelt AL. Improved heart, lung and target dose with with deep inspiration breath hold in a large clinical series of breast cancer patients. Radiotherapy and Oncology 2013; 106: 28-32.

13. Latty D, Stuart KE, Wang W. Review of deep inspiration breath-hold techniques for the breast cancer. Journal of Medical Radiation Sciences 2015; 62: 74-81.

14. Korreman SS, Pedersen AN, Nottrup TJ. Breathing adapted radiotherapy for breast cancer: Comparison of free breathing gating with breath-hold technique. Radiotherapy and Oncology 2005; 76: 311-318.

15. Hjelstuen MHB, Mjaaland I, Vikström J. Radiation during deep inspiration allows loco-regional treatment of breast and axillary-, supraclavicular- and inernal mammary lymph nodes without compromising target coverage or dose restrictions to organs at risk. Acta Oncologica 2012; 51:333344
Contato:

Laura Emília da Silva

Instituto Nacional de Câncer (INCa), Praça da Cruz

Vermelha, Rio de Janeiro (RJ)

laura.emilia.fm@gmail.com 